\title{
THE $\mathbb{F}_{2}$-COHOMOLOGY RINGS OF Sol $^{3}$-MANIFOLDS
}

\author{
J. A. HILLMAN \\ (Received 7 January 2013; accepted 13 August 2013; first published online 27 September 2013)
}

\begin{abstract}
We compute the rings $H^{*}\left(N ; \mathbb{F}_{2}\right)$ for $N$ a closed $\mathbb{S o l}^{3}$-manifold, and then determine the Borsuk-Ulam indices $\mathrm{BU}(N, \phi)$ with $\phi \neq 0$ in $H^{1}\left(N ; \mathbb{F}_{2}\right)$.
\end{abstract}

2010 Mathematics subject classification: primary 57M99.

Keywords and phrases: Borsuk-Ulam theorem, cohomology, Sol ${ }^{3}$-manifold.

The Borsuk-Ulam theorem states that any continuous function $f: S^{n} \rightarrow \mathbb{R}^{n}$ takes the same value at some antipodal pair of points. This may be put in a broader context as follows. Let $N$ be an $n$-manifold and let $N_{\phi}$ be the double cover associated to an epimorphism $\phi: \pi \rightarrow Z / 2 Z$. Let $t_{\phi}$ be the covering involution. The Borsuk-Ulam index $\operatorname{BU}(N, \phi)$ is the maximal value of $k$ such that for all maps $f: N_{\phi} \rightarrow \mathbb{R}^{k}$ there is an $x \in$ $N_{\phi}$ with $f(x)=f\left(t_{\phi}(x)\right)$. Then the Borsuk-Ulam theorem is equivalent to the assertion that $\mathrm{BU}\left(R P^{n}, \alpha\right)=n$, where $\alpha: \pi_{1}\left(R P^{n}\right) \rightarrow Z / 2 Z$ is the canonical epimorphism.

In low dimensions this invariant may be determined cohomologically, and is known for many pairs $(N, \phi)$, with $N$ a Seifert fibred 3-manifold, including all those with geometry $\mathbb{E}^{3}, \mathbb{S}^{3}, \mathbb{S}^{2} \times \mathbb{E}^{1}, \mathbb{N i l}^{3}$ or $\mathbb{H}^{2} \times \mathbb{E}^{1}[3,1]$. Here we shall determine this invariant for all such pairs with $N$ a closed $\mathbb{S o l}^{3}$-manifold. This follows easily once we know the mod-2 cohomology rings of such manifolds. We compute these using Poincaré duality and elementary properties of cup product in the low-degree cohomology of groups. (Our approach can also be applied to $\mathbb{E}^{3}$ - and $\mathbb{N i l}^{3}$-manifolds.)

\section{Sol ${ }^{3}$-manifolds and their groups}

Let $M$ be a closed $\mathbb{S o l}^{3}$-manifold. Then $\pi=\pi_{1}(M)$ has a unique maximal abelian normal subgroup $\sqrt{\pi}$, which is free abelian of rank two. (This subgroup is in fact the Hirsch-Plotkin radical [8] of $\pi$.) The quotient $\pi / \sqrt{\pi}$ is virtually $\mathbb{Z}$ (that is, has two ends), and so is an extension of $\mathbb{Z}$ or $D_{\infty}=Z / 2 Z * Z / 2 Z$ by a finite normal subgroup. The preimage of this finite normal subgroup is torsion-free, and so is either $\mathbb{Z}^{2}$ or $\mathbb{Z} \rtimes_{-1} \mathbb{Z}$ (the Klein bottle group). Since $\operatorname{Out}\left(\mathbb{Z} \rtimes_{-1} \mathbb{Z}\right)$ is finite and $\pi$ is not virtually abelian, this preimage must be $\sqrt{\pi}$. Hence $\pi / \sqrt{\pi} \cong \mathbb{Z}$ or $D_{\infty}$.

(C) 2013 Australian Mathematical Publishing Association Inc. 0004-9727/2013 \$16.00 
Suppose first that $\pi / \sqrt{\pi} \cong \mathbb{Z}$. Then $M$ is the mapping torus of a selfhomeomorphism of $T=S^{1} \times S^{1}$, and $\pi \cong \mathbb{Z}^{2} \rtimes_{\Theta} \mathbb{Z}$, where $\Theta=\left(\begin{array}{ll}a & c \\ b & d\end{array}\right) \in \operatorname{GL}(2, \mathbb{Z})$. Thus $\pi$ has a presentation

$$
\left\langle t, x, y \mid t x t^{-1}=x^{a} y^{b}, t y t^{-1}=x^{c} y^{d}, x y=y x\right\rangle .
$$

Let $\varepsilon=\operatorname{det} \Theta= \pm 1$ and $\tau=\operatorname{tr} \Theta=a+d$. Then $M$ is orientable if and only if $\varepsilon=1$, in which case $|\tau|>2$, since $\pi$ is not virtually nilpotent. Let $\theta$ be a $\operatorname{root} \operatorname{of} \operatorname{det}\left(\Theta-X I_{2}\right)=$ $X^{2}-\tau X+\varepsilon$, the characteristic polynomial of $\Theta$. Then $\theta$ is a unit in the quadratic number field $\mathbb{Q}[\theta]$, and $\sqrt{\pi}$ is isomorphic to an ideal $I$ in the ring $\mathbb{Z}[\theta]$. (The latter may not be the full ring of integers in $\mathbb{Q}[\theta]$ !)

There is a converse. Let $[I]$ denote the isomorphism class of the ideal $I$. The Galois involution of the quadratic field $\mathbb{Q}[\theta]$ acts on the $\operatorname{ring} \mathbb{Z}[\theta]$, since $\bar{\theta}=\tau-\theta \in \mathbb{Z}[\theta]$, and hence acts on the set of ideal classes.

Theorem 1.1. Let $\alpha$ be a quadratic algebraic unit which is not a root of unity, and let $J$ be a nonzero ideal in $\mathbb{Z}[\alpha]$. Let $A$ be the automorphism of $J \cong \mathbb{Z}^{2}$ given by left multiplication by $\alpha$, and let $\pi=J \rtimes_{A} \mathbb{Z}$. Then:

(1) $\pi$ is a Sol $^{3}$-group;

(2) the groups corresponding to two such pairs $(\alpha, J)$ and $(\beta, K)$ are isomorphic if and only if either $\beta=\alpha$ or $\alpha^{-1}$ and $[K]=[J]$, or $\beta=\bar{\alpha}$ or $\bar{\alpha}^{-1}$ and $[K]=\overline{[J]}$;

(3) given $\alpha$, the number of isomorphism classes of such groups $\pi$ is finite.

Proof. The group $\pi$ is the fundamental group of the mapping torus of a selfhomeomorphism of $T$. If $\alpha$ is not a root of unity then this is a $\mathrm{Sol}^{3}$-manifold.

Let

$$
\pi=\left\langle J, t \mid t j t^{-1}=\alpha j \forall j \in J\right\rangle
$$

and

$$
\widetilde{\pi}=\left\langle k, \widetilde{t} \mid \widetilde{t} \widetilde{t}^{-1}=\beta k \forall k \in K\right\rangle
$$

be two such groups. An isomorphism $f: \pi \cong \widetilde{\pi}$ restricts to an isomorphism $f_{J}: J=$ $\sqrt{\pi} \cong \sqrt{\pi}=K$. Hence it induces an isomorphism $\pi / \sqrt{\pi} \cong \widetilde{\pi} / \sqrt{\pi}$, and so $f(t)=\widetilde{t}^{\eta} k$, for some $\eta= \pm 1$ and $k \in K$. We may assume that $f(t)=\widetilde{t}$, after replacing $\beta$ by $\beta^{-1}$, if necessary. The characteristic polynomials of the automorphism of $J$ and $K$ induced by conjugation by $t$ and $\widetilde{t}$ (respectively) must then agree. Thus either $\beta=\alpha$ and $f_{J}$ is an isomorphism of $\mathbb{Z}[\alpha]$-modules, or $\beta=\bar{\alpha}$ and $\overline{f_{J}}: J \rightarrow \bar{K}$ is an an isomorphism of $\mathbb{Z}[\alpha]$-modules. The converse is similarly straightforward.

The group $\pi$ is determined up to a finite ambiguity by $\alpha$ (equivalently, by the polynomial $t^{2}-\tau t+\varepsilon$ ), since $\mathbb{Z}[\alpha]$ has finitely many ideal classes, by the JordanZassenhaus theorem.

If $\pi / \sqrt{\pi} \cong D_{\infty}$ then $\pi \cong B *_{T} C$, where $B$ and $C$ are torsion-free, $T \cong \mathbb{Z}^{2}$ and $[B$ : $T]=[C: T]=2$. Thus $M$ is the union of two twisted $I$-bundles. Since $\beta_{1}(\pi ; \mathbb{Q})=0$ and $\chi(M)=0, M$ is orientable, and so $B$ and $C$ must be copies of the Klein bottle group. Hence $M$ is the union of two copies of the mapping cylinder of the double 
cover of the Klein bottle. The double cover of $M$ corresponding to the preimage of $\sqrt{D_{\infty}}$ in $\pi$ is a mapping torus.

In particular, $\pi$ has a presentation

$$
\left\langle u, v, y, z \mid u y u^{-1}=y^{-1}, v z v^{-1}=z^{-1}, y z=z y, v^{2}=u^{2 a} y^{b}, z=u^{2 c} y^{d}\right\rangle,
$$

where $\left(\begin{array}{ll}a & c \\ b & d\end{array}\right) \in \operatorname{GL}(2, \mathbb{Z})$ corresponds to the identification of $\sqrt{C}$ with $T=\sqrt{B}$. This presentation simplifies immediately to

$$
\left\langle u, v, y \mid u y u^{-1}=y^{-1}, v^{2}=u^{2 a} y^{b}, v u^{2 c} y^{d} v^{-1}=u^{-2 c} y^{-d}\right\rangle .
$$

Hence $\pi^{\mathrm{ab}} \cong Z / 4 c Z \oplus Z / 4 Z$ if $b$ is odd, and $\pi^{\mathrm{ab}} \cong Z / 4 c Z \oplus(Z / 2 Z)^{2}$ if $b$ is even. Let $x=u^{2}$. Then conjugation by $u v$ acts on $\langle x, y\rangle \cong \mathbb{Z}^{2}$ via $\Psi=\eta\left(\begin{array}{cc}a d+b c & 2 a c \\ 2 b d & a d+b c\end{array}\right)$, where $\eta=a d-b c= \pm 1$. Hence $\operatorname{det} \Psi=1, \Psi \equiv I_{2}(\bmod 2)$ and $\operatorname{tr} \Psi \equiv 2(\bmod 4)$. (These conditions are not independent, for if $\Psi=I_{2}+2 N$ then $\operatorname{tr} \Psi=2+2 \operatorname{tr} N$ and $\operatorname{det} \Psi \equiv$ $1+2 \operatorname{tr} N(\bmod 4)$, so $\operatorname{tr} N$ is even and $\operatorname{tr} \Psi \equiv 2(\bmod 4)$ if also $\operatorname{det} \Psi=1$.) Moreover, abcd $\neq 0$, since $M$ is not flat.

Conversely, any $\left(\begin{array}{ll}a & c \\ b & d\end{array}\right) \in \mathrm{GL}(2, \mathbb{Z})$ with $a b c d \neq 0$ gives rise to such a $\mathbb{S o l}^{3}$-manifold, for then $|\operatorname{tr} \Psi|=2|a d+b c| \geq 6$. Moreover, suppose that $P=\left(\begin{array}{cc}2 k+1 & 2 m \\ 2 n & 2 k+1\end{array}\right) \in \operatorname{SL}(2, \mathbb{Z})$, where $m n \neq 0$. Then $k(k+1)=m n$, and so we may write $m=m_{1} m_{2}$ and $n=n_{1} n_{2}$, with $k=m_{1} n_{1}$ and $k+1=m_{2} n_{2}$. The $\mathbb{S o l}^{3}$-rational homology sphere corresponding to $\left(\begin{array}{cc}m_{1} & -m_{2} \\ -n_{2} & n_{1}\end{array}\right) \in \mathrm{GL}(2, \mathbb{Z})$ is doubly covered by the mapping torus associated to $P$.

The above matrix calculations show that a quadratic unit $\alpha$ is realised by such a $\mathrm{Sol}^{3}$-manifold if and only if $\alpha \bar{\alpha}=1,|\alpha+\bar{\alpha}|>2$ and $\alpha+\bar{\alpha} \equiv 2(\bmod 4)$. Determining the possible ideal classes represented by $\sqrt{\pi}$ is more complicated.

THeOREM 1.2. Let $\alpha$ be a quadratic unit which is not a root of unity, and let $J$ be a nonzero ideal in $\mathbb{Z}[\alpha]$. Let $A$ be the automorphism of $J \cong \mathbb{Z}^{2}$ given by left multiplication by $\alpha$, and let $\kappa=J \rtimes_{A} \mathbb{Z}$. Then $\kappa$ is a subgroup of index 2 in a Sol $^{3}$-group $\pi$ with $\pi / \sqrt{\pi} \cong$ $D_{\infty}$ if and only if $\alpha \bar{\alpha}=1, \alpha \equiv 1(\bmod 2 \mathbb{Z}[\alpha])$ and there are $\lambda, \mu \neq 0 \in \mathbb{Z}[\alpha]$ and $v, w \in J$ such that $\lambda \bar{J}=\mu J, \lambda \bar{v}=\mu v$ and $\lambda \bar{w}=\bar{\alpha} \mu w$, but $\bar{\lambda} v \neq \bar{\lambda} j+\bar{\mu} \bar{j}$ and $\bar{\lambda} w \neq \bar{\lambda} j+\alpha \bar{\mu} \bar{j}$ for any $j \in J$.

Given $\alpha$, the number of isomorphism classes of such groups $\pi$ is finite.

Proof. Suppose that $\pi=\langle\kappa, u\rangle$ with $\pi / \sqrt{\pi} \cong D_{\infty}$ and $[\pi: \kappa]=2$, and that $t \in \kappa$ generates $\kappa(\bmod \sqrt{\pi})$. Then $t^{-1}$ is conjugate to $t$, and so $A$ and $A^{-1}$ have the same characteristic polynomial. Since $\operatorname{tr} A \neq 0, \alpha \bar{\alpha}=\operatorname{det} A=1$.

Let $B(j)=u j u^{-1}$ and $f(j)=\overline{B(j)}$, for all $j \in J$. Then $B$ is an isomorphism of groups and $f: J \rightarrow \bar{J}$ is an isomorphism of $\mathbb{Z}[\alpha]$-modules. Let $v=u^{2}$ and $w=(t u)^{2}$. Then $B^{2}=(A B)^{2}=I, B v=v$ and $A B w=w$. Since $A$ has infinite order, $B \neq I$, and so $\operatorname{det} B=-1$. Moreover, $B \equiv A B \equiv I_{2}(\bmod 2)$, since $\langle J, u\rangle$ and $\langle J, t u\rangle \cong \pi_{1}(K b)$. Therefore $A \equiv I_{2}(\bmod 2)$ also, and so $\alpha \equiv 1(\bmod 2 \mathbb{Z}[\alpha])$.

Since $\pi$ is torsion-free, $(u j)^{2}$ and $(t u j)^{2}$ are nontrivial, for all $j \in J$. Equivalently, $v \notin(I+B) J$ and $w \notin(I+A B) J$. 
The isomorphism $f$ extends to an automorphism $f_{\mathbb{Q}}=i d_{\mathbb{Q}} \otimes f$ of $\mathbb{Q}[\alpha]$, as a vector space over itself. We may write $f_{\mathbb{Q}}(1)=\mu / \lambda$, for some nonzero $\lambda, \mu \in \mathbb{Z}[\alpha]$. (Note that $\mu \bar{\mu} / \lambda \bar{\lambda}=\operatorname{det} f_{\mathbb{Q}}=-\operatorname{det} B=1$.) Then $\lambda f(j)=\mu j$, for all $j \in J$, since $\mathbb{Z}[\alpha]$ is an integral domain. The linear conditions on $v$ and $w$ become $\lambda \bar{v}=\mu v$ and $\lambda \bar{w}=\bar{\alpha} \mu w$, while $\bar{\lambda} v \neq \bar{\lambda} j+\bar{\mu} \bar{j}$ and $\bar{\lambda} w \neq \bar{\lambda} j+\alpha \bar{\mu} \bar{j}$ for any $j \in J$.

Conversely, suppose that these conditions hold. Let $B j=\overline{(\mu / \lambda) j}$, for all $j \in J$, and let $\pi$ be the group with presentation

$$
\left\langle\kappa, u \mid u^{2}=v, u t u^{-1}=t^{-1} w v^{-1}, u j u^{-1}=B j \forall j \in J\right\rangle .
$$

Then $\pi$ is torsion-free and has $\kappa$ as a subgroup of index 2. and so is a Sol $^{3}$-group. Clearly $\pi / \sqrt{\kappa} \cong D_{\infty}$, and so $\sqrt{\kappa} \leq \sqrt{\pi} \leq \kappa$. Hence $\sqrt{\pi}=\sqrt{\kappa}$ and $\pi / \sqrt{\pi} \cong D_{\infty}$.

Since $\kappa$ has trivial centre the extensions of $Z / 2 Z$ by $\kappa$ are determined by the image in $\operatorname{Out}(\kappa)$ of the action of $Z / 2 Z$ on $\kappa$. Since there are finitely many groups $\kappa$ realising $\alpha$, by Theorem 1.1, and $\operatorname{Out}(\kappa)$ is finite, by [5, Theorem 8.10], there are finitely many such groups $\pi$.

In particular, the ideal class $[J]$ must be fixed by the Galois involution. For example, if $\alpha \bar{\alpha}=1$ and $\alpha \equiv 1(\bmod 2 \mathbb{Z}[\alpha])$ then $J=\mathbb{Z}[\alpha], v=1$ and $w=\alpha$ satisfy the other conditions, with $\lambda=\mu=1$.

Note that if $\alpha$ is a quadratic unit such that $\alpha \bar{\alpha}=1$ and $\delta=\alpha-1 \in 2 \mathbb{Z}[\alpha]$ then $\bar{\delta}=-\alpha^{-1} \delta \in 2 \mathbb{Z}[\alpha]$ also, and so $\alpha+\bar{\alpha}=2-\delta \bar{\delta} \equiv 2(\bmod 4)$. (This is equivalent to an earlier matrix argument.)

Every subgroup of finite index in $\pi$ can be generated by three elements, while proper subgroups of infinite index need at most two generators. If a nontrivial normal subgroup $N$ has infinite index in $\pi$ then it has Hirsch length at most 2. Hence it is abelian, and so has finite index in $\sqrt{\pi}$. Thus proper quotients of a Sol $^{3}$-group $\pi$ either have two ends or are finite.

\section{The mod-2 cohomology ring}

Martins has constructed an explicit free resolution $P_{*} \rightarrow \mathbb{Z}$ of the augmentation $\mathbb{Z}[\pi]$-module, and a diagonal approximation $\Delta: P_{*} \rightarrow P_{*} \otimes P_{*}$, which he used to compute the integral and mod- $p$ cohomology rings for semidirect products $\pi \cong \mathbb{Z}^{2} \rtimes_{\Theta}$ $\mathbb{Z}$ with $\Theta \in \mathrm{GL}(2, \mathbb{Z})[6]$.

We shall take a somewhat different approach, first computing cup products into $H^{2}\left(\pi ; \mathbb{F}_{2}\right)$ and then using Poincaré duality. Our strategy in determing relations in $H^{2}\left(\pi ; \mathbb{F}_{2}\right)$ shall be to use restrictions to subgroups (such as $\sqrt{\pi}$ ) and epimorphisms to quotient groups (such as $\pi / \sqrt{\pi}$ or small finite 2-groups), with known cohomology rings.

We shall usually write $H_{*}(X)$ and $H^{*}(X)$ for the homology and cohomology of a space or group $X$, with coefficients $\mathbb{F}_{2}$, and denote the cup product by juxtaposition. In each case considered below, the given generators for a group $G$ represent a basis for $H_{1}(G)$, and we shall use the corresponding Kronecker dual bases for $H^{1}(G)=$ $\operatorname{Hom}\left(H_{1}(G), \mathbb{F}_{2}\right)$. 
Lemma 2.1. Let $w=w_{1}(\pi)$. Then $w \alpha \beta=\alpha^{2} \beta+\alpha \beta^{2}$, for all $\alpha, \beta \in H^{1}(\pi)$. In particular, if $w=0$ then $\alpha^{2} \beta=\alpha \beta^{2}$ and $(\alpha+\beta)^{3}=\alpha^{3}+\beta^{3}$.

Proof. The first assertion follows from the Wu relation $S q^{1} z=w z$, for all $z \in H^{n-1}(X)$, which holds for any $P D_{n}$-complex $X$. The second follows easily.

If $G$ is a group let $X^{n}(G)=\left\langle g^{n} \mid g \in G\right\rangle$ be the subgroup generated by all $n$th powers. The next lemma is a refinement of [4, Theorem 2] (which is restated here as part (1) of the lemma).

Lemma 2.2. Let $G$ be a group, and $\rho, \phi, \psi \in H^{1}(G)$. Let $K=\operatorname{Ker}(\rho)$ and $L=K \cap$ $\operatorname{Ker}(\phi)$. Then:

(1) the kernel of cup product from the symmetric product $\odot^{2} H^{1}(G)$ to $H^{2}(G)$ is the dual of $X^{2}(G) / X^{4}(G)\left[G, X^{2}(G)\right]$;

(2) the canonical projections induce isomorphisms

$$
H^{1}\left(G / X^{2}(K)\right) \cong H^{1}\left(G / X^{2}(L)\right) \cong H^{1}\left(G / X^{4}(G)\right) \cong H^{1}(G)
$$

(3) $\rho \phi=0$ in $H^{2}(G)$ if and only if $\rho \phi=0$ in $H^{2}\left(G / X^{2}(K)\right)$;

(4) $\phi^{2}=\rho \phi+\rho \psi$ in $H^{2}(G)$ if and only if $\phi^{2}=\rho \phi+\rho \psi$ in $H^{2}\left(G / X^{2}(L)\right)$.

Proof. Part (1) is [4, Theorem 2], while part (2) is clear.

If $\phi \psi=0$ in $H^{2}(G)$ then there is a 1-cochain $F: G \rightarrow \mathbb{F}_{2}$ such that $\phi(g) \psi(h)=$ $\delta F(g, h)=F(g h)+F(g)+F(h)$, for all $g, h \in G$. Part (3) follows easily, since $F$ restricts to a homomorphism on $K$, and is constant on cosets of $X^{2}(K)$.

Part (4) is similar.

In many of the cases considered here, the coefficients in the linear relations determining the kernel of cup product may be found by restricting to 2-generator subgroups. However, this is not always enough to determine the triple products in $H^{3}(\pi)$.

Lemma 2.3. Let $\{T, Y\}$ be the basis for $H^{1}\left(D_{8}\right)$ corresponding to the presentation $D_{8}=\left\langle t, y \mid t^{2}=y^{4}=1, t y t^{-1}=y^{-1}\right\rangle$. Then $(T+Y) Y=0$ in $H^{2}\left(D_{8}\right)$.

Proof. Let $D_{\infty}$ have the presentation $\left\langle u, v \mid u^{2}=v^{2}=1\right\rangle$, and let $U, V$ be the dual basis for $H^{1}\left(D_{\infty}\right)$. Then $H^{*}\left(D_{\infty}\right)=\mathbb{F}_{2}[U, V] /(U V)$. Let $f: D_{\infty} \rightarrow D_{8}$ be the epimorphism given by $f(u)=t$ and $f(v)=t y$. Then $f$ induces an isomorphism $D_{\infty} / X^{4}\left(D_{\infty}\right) \cong D_{8}$, so $H^{2}(f)$ is injective. Since $f^{*} U=T+Y$ and $f^{*} V=Y$, we see that $(T+Y) Y=0$ in $H^{2}\left(D_{8}\right)$.

Let $E$ be the 'almost extraspecial' 2-group with presentation

$$
\left\langle t, u, v \mid t^{2}=1, u^{2}=v^{2}, t u t^{-1}=u^{-1}, t v=v t, u v=v u\right\rangle .
$$

Lemma 2.4. Let $\{T, U, V\}$ be the basis for $H^{1}(E)$ corresponding to the above presentation. Then $T U+U^{2}+V^{2}=0$ in $H^{2}(E)$. 
Proof. Since $X^{2}(E) \cong Z / 2 Z$, the kernel of cup product from $\odot^{2} H^{1}(G)$ to $H^{2}(G)$ has dimension one [4]. Thus there is a unique nontrivial linear relation $a T^{2}+b U^{2}+$ $c V^{2}+d T U+e T V+f U V=0$ in $H^{2}(E)$. The coefficients can be determined by restriction to the subgroups $\langle t\rangle \cong Z / 4 Z,\langle t, u\rangle \cong D_{8},\langle t, v\rangle \cong Z / 4 Z \oplus Z / 2 Z$, and $\langle u, v\rangle \cong$ $Z / 4 Z \oplus Z / 2 Z$.

\section{Mapping tori}

Suppose that $\pi \cong \mathbb{Z}^{2} \rtimes_{\Theta} \mathbb{Z}$, where $\Theta=\left(\begin{array}{ll}a & c \\ b & d\end{array}\right) \in \mathrm{GL}(2, \mathbb{Z})$. Let $\varepsilon=a d-b c= \pm 1$ and $\tau=a+d$. Let $\Delta_{1}=\operatorname{det}\left(\Theta-I_{2}\right)=1-\tau+\varepsilon$ and $\Delta_{2}=(a-1, b, c, d-1)$ be the elementary divisors of $\Theta-I_{2}$. Then $\Delta_{2}^{2}$ divides $\Delta_{1}$, and

$$
\pi^{\mathrm{ab}} \cong \mathbb{Z} \oplus Z /\left(\Delta_{1} / \Delta_{2}\right) Z \oplus Z / \Delta_{2} Z
$$

Let $\beta=\beta_{1}\left(\pi ; \mathbb{F}_{2}\right)$. Then $1 \leq \beta \leq 3$, and $\beta_{2}\left(\pi ; \mathbb{F}_{2}\right)=\beta$, by Poincaré duality. Let $\rho: \pi \rightarrow Z / 2 Z$ be the unique epimorphism which factors through $\pi / \sqrt{\pi} \cong \mathbb{Z}$. If $\pi$ is nonorientable then $\rho=w_{1}(M)$, and $K=\pi^{+}$, the maximal orientable subgroup of $\pi$.

(1) If $\tau$ is odd then $\Delta_{1}$ is odd and $\pi^{\mathrm{ab}} \cong \mathbb{Z} \oplus o d d$. In this case $\rho$ is the unique epimorphism from $\pi$ to $Z / 2 Z$, and

$$
H^{*}(\pi) \cong \mathbb{F}_{2}[\rho, \Xi] /\left(\rho^{2}, \Xi^{2}\right),
$$

where $\Xi$ has degree two, by Poincaré duality.

(2) If $\tau \equiv \varepsilon-1(\bmod 4)$ then $\pi^{\mathrm{ab}} \cong \mathbb{Z} \oplus Z / 2 Z \oplus$ odd, and $\beta=2$. Hence $H^{1}(\pi)=$ $\langle\rho, \sigma\rangle$, where $\sigma$ does not factor through $Z / 4 Z$. Moreover, if $G=\pi / X^{4}(\pi)$ then $X^{2}(G) \cong$ $(Z / 2 Z)^{2}$ is central in $G$. Thus $\rho^{2}=\rho \sigma=0$, by Lemma 2.2, while $\sigma^{2} \neq 0$. Hence $H^{2}(\pi)=\left\langle\sigma^{2}, \Xi\right\rangle$, for some $\Xi$ of degree two. Duality then implies that $\sigma^{3}=\rho \Xi \neq 0$. We may assume also that $\sigma \Xi=0$, and so

$$
H^{*}(\pi) \cong \mathbb{F}_{2}[\rho, \sigma, \Xi] /\left(\rho^{2}, \rho \sigma, \sigma \Xi, \rho \Xi+\sigma^{3}, \Xi^{2}\right) .
$$

(3) If $\tau \equiv \varepsilon+1(\bmod 4)$ and $\Delta_{2}$ is odd then $\pi^{\mathrm{ab}} \cong \mathbb{Z} \oplus Z / 2^{k} Z \oplus$ odd, for some $k \geq 2$. Hence $H^{1}(\pi)=\langle\rho, \sigma\rangle$, where $\sigma^{2}=\rho^{2}=0$. Since $\rho \sigma=0$, by the nondegeneracy of Poincaré duality,

$$
H^{*}(\pi) \cong \mathbb{F}_{2}[\rho, \sigma, \Xi, \Omega] /\left(\rho^{2}, \rho \sigma, \sigma^{2}, \rho \Omega, \sigma \Xi, \rho \Xi+\sigma \Omega, \Xi^{2}, \Omega^{2}, \Xi \Omega\right),
$$

where $\Xi$ and $\Omega$ have degree two.

In all the remaining cases $\beta=3$. For if $\tau \equiv \varepsilon+1(\bmod 4)$ and $\Delta_{2}$ is even then $a$ and $d$ are odd and $b$ and $c$ are even. Hence $\Delta_{1}=2^{k} q$ and $\Delta_{2}=2^{\ell} q^{\prime}$, where $0<\ell \leq k / 2$ and $q, q^{\prime}$ are odd. In this case $\pi^{\mathrm{ab}} \cong \mathbb{Z} \oplus Z / 2^{k-\ell} Z \oplus Z / 2^{\ell} Z \oplus$ odd, so the images of $\{t, x, y\}$ form a basis for $H_{1}(\pi)$. Let $\{\rho, \sigma, \psi\}$ be the dual basis, so that

$$
\sigma(x)=\psi(y)=1 \quad \text { and } \quad \sigma(t)=\sigma(y)=\psi(t)=\psi(x)=0 .
$$

If $G=\pi / X^{4}(\pi)$ then $X^{2}(G)=\left\langle t^{2}, x^{2}, y^{2}\right\rangle \cong(Z / 2 Z)^{3}$ is central in $G$, so the kernel of cup product from $\odot^{2} H^{1}(\pi)$ to $H^{2}(\pi)$ has rank three. It then follows from Poincaré 
duality that $H^{*}(\pi)$ is generated as a ring by $H^{1}(\pi)$. In each case, $\rho \sigma^{2}=\rho \rho \sigma=0$ and $\rho \psi^{2}=\rho \rho \psi=0$, by Lemma 2.1. Hence $\rho \sigma \psi \neq 0$, by the nondegeneracy of Poincaré duality. It then follows easily that $\rho \sigma, \rho \psi$ and $\sigma \psi$ are linearly independent, and so form a basis for $H^{2}(\pi)$. We may write

$$
\sigma^{2}=m \rho \sigma+n \rho \psi+p \sigma \psi \quad \text { and } \quad \psi^{2}=q \rho \sigma+r \rho \psi+s \sigma \psi,
$$

for some $m, \ldots, s$. On restricting to $\sqrt{\pi}$, we see that $p=s=0$, since $\left.\sigma^{2}\right|_{\sqrt{\pi}}=\left.\psi^{2}\right|_{\sqrt{\pi}}=0$ and $\left.\rho\right|_{\sqrt{\pi}}=0$, while $\left.\sigma \psi\right|_{\sqrt{\pi}} \neq 0$. Since $\rho \sigma^{2}=\rho^{2} \sigma=\rho \psi^{2}=\rho^{2} \psi=0$, taking cup products with $\sigma$ and $\psi$ gives

$$
\sigma^{3}=n \rho \sigma \psi, \quad \sigma^{2} \psi=m \rho \sigma \psi, \quad \psi^{3}=q \rho \sigma \psi \quad \text { and } \quad \sigma \psi^{2}=r \rho \sigma \psi .
$$

(4) If $\ell \geq 2$ then $a \equiv d \equiv 1$ and $b, c \equiv 0(\bmod 4)$, so $\varepsilon \equiv 1(\bmod 4)$ also, that is, $\pi$ is orientable. In this case $\sigma^{2}=\psi^{2}=\rho^{2}=0$, and so

$$
H^{*}(\pi) \cong \mathbb{F}_{2}[\rho, \sigma, \psi] /\left(\rho^{2}, \sigma^{2}, \psi^{2}\right) .
$$

Suppose now that $\ell=1$.

(5) If $\pi$ is orientable and $\Delta_{1} \equiv 0(\bmod 8)$ we may assume that one of $\sigma, \psi$ or $\sigma+\psi$ factors through $Z / 4 Z$. Thus either $\sigma^{2}=0, \psi^{2}=0$ or $\sigma^{2}=\psi^{2}$. We may assume that $\sigma^{2} \neq 0$. Then $\rho \sigma^{2}=\rho^{2} \sigma=0$ and $\psi \sigma^{2}=\psi^{2} \sigma=0$, and so $\sigma^{3} \neq 0$, by the nonsingularity of Poincaré duality. Hence

$$
H^{*}(\pi) \cong \mathbb{F}_{2}[\rho, \sigma, \psi] /\left(\rho^{2}, \rho \psi+\sigma^{2}, \psi^{2}\right) .
$$

In this case we see that $\phi^{3}=0$ if and only if $\phi^{2}=0$.

If $\pi$ is orientable and $\Delta_{1} \equiv 4(\bmod 8)$ then $\tau \equiv 6(\bmod 8)$ and $a, d$ are odd, and so $a \equiv d(\bmod 4)$. In this case $\psi^{2} \neq 0$ and $(\sigma+\psi)^{2} \neq 0$ also, and so $\sigma^{2}=m \rho \sigma+n \rho \psi$ and $\psi^{2}=q \rho \sigma+r \rho \psi$ are linearly independent. Hence $m r+n q=1$ in $\mathbb{F}_{2}$. Since $w=0$, $\sigma^{2} \psi=\sigma \psi^{2}$ and so $m=r$.

(6) Suppose first that $a \equiv 1(\bmod 4)$. Then $b c \equiv 4(\bmod 8)$, and so $b \equiv c \equiv 2(\bmod 4)$. Let $L_{\phi}=\operatorname{Ker}(\rho) \cap \operatorname{Ker}(\phi)$. Then $\pi / X^{2}\left(L_{\phi}\right)$ has a presentation

$$
\left\langle t, x, y \mid t^{4}=x^{4}=y^{2}=1, t x=x t, t y t^{-1}=x^{2} y, x y=y x\right\rangle .
$$

Let $J=\langle t, x\rangle \cong(Z / 4 Z)^{2}$. Then $\left.\sigma^{2}\right|_{J}=\left.\rho \psi\right|_{J}=0$, while $\left.\rho \sigma\right|_{J} \neq 0$. Applying part (3) of Lemma 2.2, we see that $m=0$, and so $\sigma^{2}=\rho \psi$ and $\psi^{2}=\rho \sigma$. (Note, however, that Lemma 2.2 does not assert that the relation $\psi^{2}=q \rho \sigma+r \rho \psi$ also holds in $\pi / X^{2}\left(L_{\phi}\right)$ ! For this, we could use $L_{\psi}=\operatorname{Ker}(\rho) \cap \operatorname{Ker}(\psi)$ instead.) Hence

$$
H^{*}(\pi) \cong \mathbb{F}_{2}[\rho, \sigma, \psi] /\left(\rho^{2}, \rho \psi+\sigma^{2}, \rho \sigma+\psi^{2}\right) .
$$

In particular, $\sigma^{3}=\psi^{3}=(\rho+\sigma)^{3}=(\rho+\psi)^{3} \neq 0$.

If $a \equiv-1(\bmod 4)$ then $b c \equiv 0(\bmod 8)$. If, say, $b \equiv 2(\bmod 4)($ so $c \equiv 0(\bmod 4))$ then the change of basis $x^{\prime}=x, y^{\prime}=x y$ reduces this case to the one just considered. In terms of the given basis,

$$
H^{*}(\pi) \cong \mathbb{F}_{2}[\rho, \sigma, \psi] /\left(\rho^{2}, \rho \sigma+\sigma^{2}, \rho \psi+\sigma^{2}+\psi^{2}\right) .
$$


In this case $\sigma^{3} \neq 0$, but $\psi^{3}=0$. A similar result holds if $b \equiv 0(\bmod 4)$ and $c \equiv$ $2(\bmod 4)$.

(7) If, however, $a \equiv-1(\bmod 4)$ and $b \equiv c \equiv 0(\bmod 4)$ then $\pi / X^{4}(\pi)$ has a presentation

$$
\left\langle t, x, y \mid t^{4}=x^{4}=y^{4}=1, t x t^{-1}=x^{-1}, t y t^{-1}=y^{-1}, x y=y x\right\rangle .
$$

In this case $J=\langle t, x\rangle$ is nonabelian, and $\left.\sigma^{2}\right|_{J} \neq 0$, while $\left.\rho \psi\right|_{J}=0$. Hence we must have $m=r=1$. It is clear from the symmetry of the presentation for $\pi / X^{4}(\pi)$ that we must also have $n=q$ in this case, and so $n=q=0$. Thus

$$
H^{*}(\pi) \cong \mathbb{F}_{2}[\rho, \sigma, \psi] /\left(\rho^{2}, \rho \sigma+\sigma^{2}, \rho \psi+\psi^{2}\right) .
$$

We now find that $\phi^{3}=0$ for all $\phi \in H^{1}(\pi)$.

If $\ell=1$ and $M$ is nonorientable then $a$ and $d$ are odd, and $\Delta_{1}=-a-d \equiv 0(\bmod 4)$. In this case $\rho=w_{1}(M)$, and so $\sigma^{2} \psi+\sigma \psi^{2}=\rho \sigma \psi \neq 0$, by Lemma 2.1. After swapping $x$ and $y$, if necessary, we may assume that $a \equiv 1(\bmod 4)$.

(8) If $b c \equiv 0(\bmod 8)$ then, after a further change of basis of the form $x^{\prime}=x, y^{\prime}=x y$ or $x^{\prime}=x y, y^{\prime}=y$, if necessary, we may assume that $b \equiv c \equiv 0(\bmod 4)$. Then $\sigma^{2}=0$, and $\pi /\left\langle\left\langle t^{2}, x, y^{4}\right\rangle\right\rangle \cong D_{8}$, so $(\rho+\psi) \psi=0$ also. Hence

$$
H^{*}(\pi) \cong \mathbb{F}_{2}[\rho, \sigma, \psi] /\left(\rho^{2}, \sigma^{2}, \rho \psi+\psi^{2}\right) .
$$

In particular, $(\sigma+\psi)^{3}=(\rho+\sigma+\psi)^{3} \neq 0$, and all other classes have cube 0 . In terms of the given bases, the other cases are as follows.

If $b \equiv 0$ and $c \equiv 2(\bmod 4)$ then

$$
H^{*}(\pi) \cong \mathbb{F}_{2}[\rho, \sigma, \psi] /\left(\rho^{2}, \sigma^{2}+\psi^{2}, \rho \psi+\psi^{2}, \sigma^{2} \psi\right) .
$$

Here $\sigma^{3}=(\rho+\sigma)^{3} \neq 0$ and all other classes have cube 0 .

If $b \equiv 2$ and $c \equiv 0(\bmod 4)$ then

$$
H^{*}(\pi) \cong \mathbb{F}_{2}[\rho, \sigma, \psi] /\left(\rho^{2}, \sigma^{2}, \psi^{2}+\rho \sigma+\rho \psi\right) .
$$

Here $\psi^{3}=(\rho+\psi)^{3} \neq 0$ and all other classes have cube 0 .

(9) If $b \equiv c \equiv 2(\bmod 4)$ then $\sigma^{2}$ and $\psi^{2}$ are linearly independent. There are three distinct epimorphisms from $\pi$ to the almost extraspecial group $E$, given by $f(x)=u^{-1} v$, $f(y)=u ; g(x)=v, g(y)=u v^{-1} ;$ and $h(x)=v, h(y)=u$. Using these epimorphisms to pull back the relation given in Lemma 2.3, we find that

$$
H^{*}(\pi) \cong \mathbb{F}_{2}[\rho, \sigma, \psi] /\left(\rho^{2}, \sigma^{2}+\rho \psi, \psi^{2}+\rho \sigma+\rho \psi\right) .
$$

In particular, every epimorphism $\phi \neq \rho$ has nonzero cube. 


\section{Unions of twisted $I$-bundles}

Suppose that $\pi / \sqrt{\pi} \cong D_{\infty}$. Then $\pi$ is orientable, and has a presentation

$$
\left\langle u, v, y \mid u y u^{-1}=y^{-1}, v^{2}=u^{2 a} y^{b}, v u^{2 c} y^{d} v^{-1}=u^{-2 c} y^{-d}\right\rangle,
$$

where $a d-b c= \pm 1$ and $a b c d \neq 0$. Let $B=\langle u, y\rangle$ and $C=\left\langle v, u^{2 c} y^{d}\right\rangle$.

If $b$ is odd then $\pi^{\mathrm{ab}} \cong Z / 4 c Z \oplus Z / 4 Z$, where the summands are generated by $u$ and $u^{-a} v$, respectively. Let $U(u)=V(v)=1, U(v)=a$ and $V(u)=0$. Then

$$
H^{*}(\pi) \cong \mathbb{F}_{2}[U, V, \Xi, \Omega] /\left(U^{2}, U V, V^{2}, U \Xi+V \Omega, \Xi^{2}, \Omega^{2}, \Xi \Omega\right),
$$

where $\Xi$ and $\Omega$ have degree two.

If $b$ is even then $\pi^{\mathrm{ab}} \cong Z / 4 c Z \oplus(Z / 2 Z)^{2}$ and the images of $u, v$ and $y$ represent a basis for $H_{1}(\pi)$. Let $\{U, V, Y\} \in H^{1}(\pi)$ be the dual basis. Then $U^{2}, V^{2}$ and $Y^{2}$ are all nonzero, but $W=U+V$ lifts to a homomorphism from $\pi$ to $Z / 4 Z$, and so $W^{2}=0$. Hence $U^{2}=V^{2}$. Since $U$ and $V$ are induced from classes in $H^{1}\left(D_{\infty}\right)$ we have $U V=0$. We also have $\left.U Y\right|_{B}=\left.Y^{2}\right|_{B}$ and $\left.V Y\right|_{C}=\left.Y^{2}\right|_{C}$, while $\left.U\right|_{C},\left.V\right|_{B},\left.U^{2}\right|_{B}$ and $\left.V^{2}\right|_{C}$ are all 0 .

Suppose that $p U^{2}+q Y^{2}+r U Y+s V Y=0$ in $H^{2}(\pi)$. On restricting to the subgroups $B$ and $C$, we find that $q+r=q+s=0$. Since $U^{2} \neq 0$ we must have $q=$ $r=s=1$. Multiplying by $U$ and $V$, we find that $U Y^{2}+U^{2} Y=0$ and $V Y^{2}+V^{2} Y=0$. Poincaré duality for $\pi$ now implies that $\left\{U^{2}, Y^{2}, U Y\right\}$ is a basis for $H^{2}(\pi)$, while $U Y^{2}=$ $U^{2} Y=V Y^{2}$ generates $H^{3}(\pi)$. We see also that $U^{3}=U^{2} V=U V^{2}=V^{3}=(U+V)^{3}=0$, while $(U+Y)^{3}=(V+Y)^{3}=(U+V+Y)^{3}=Y^{3}$.

If $b \equiv 0(\bmod 4)$ then $G=\pi /\left\langle\left\langle u v, u^{2}, y^{4}\right\rangle\right\rangle \cong D_{8}$. Hence $(U+V+Y) Y=0$ in $H^{3}(\pi)$. It follows easily that $Y^{3}=0$, and so all cubes are 0 in $H^{3}(\pi)$.

If $b \equiv 2(\bmod 4)$ then $\pi /\left\langle\left\langle u^{2},(u v)^{2}, v^{4}, y^{4}\right\rangle\right\rangle$ has a presentation

$$
\left\langle u, v, y \mid u^{2}=(u v)^{2}=v^{4}=1, u y u^{-1}=v y v^{-1}=y^{-1}, v^{2}=y^{2}\right\rangle .
$$

Hence there is an epimorphism $f: \pi \rightarrow E$, given by $f(u)=t, f(v)=u$ and $f(y)=$ $u^{-1} t^{-1} v$. Since $f^{*} T=U+Y, f^{*} U=V+Y, f^{*} V=Y$ and $U V=0$, it follows from Lemma 2.4 that $U Y+V Y+V^{2}+Y^{2}=0$ in $H^{2}(\pi)$. Multiplying by $Y$, we find that $U Y^{2}+Y^{3}=0$ and so $Y^{3} \neq 0$. In this case, only the cubes induced from $H^{*}(\pi / \sqrt{\pi})$ are zero.

\section{The Borsuk-Ulam index}

We may identify an epimorphism $\phi$ with a nonzero class in $H^{1}\left(N ; \mathbb{F}_{2}\right)$. Then $\mathrm{BU}(N, \phi)=1$ if and only if $\phi$ lifts to an integral class $\Phi \in H^{1}(N ; \mathbb{Z})$, while $\mathrm{BU}(N, \phi)=$ $n$ if and only if $\phi^{n} \neq 0$ in $H^{n}\left(N ; \mathbb{F}_{2}\right)$. In general, $1 \leq \mathrm{BU}(N, \phi) \leq n$. See [3]. When $n=3$ the remaining possibility is that $\mathrm{BU}(M, \phi)=2$ if and only if $\phi^{2}=0$ but $\phi$ is not the reduction of an integral class.

Suppose first that $\pi / \sqrt{\pi} \cong \mathbb{Z}$. Then the following results are immediate from Section 3. 
(1) If $\rho: \pi \rightarrow Z / 2 Z$ is the unique epimorphism which factors through $\pi / \sqrt{\pi} \cong \mathbb{Z}$ then $\mathrm{BU}(M, \rho)=1$.

(2) If $\tau \equiv \varepsilon-1(\bmod 4)$ then $\operatorname{BU}(M, \phi)=3$ for all $\phi \neq \rho$.

(3) If $\tau \equiv \varepsilon+1(\bmod 4)$ and either $\Delta_{2}$ is odd or $a \equiv d \equiv 1(\bmod 4)$ and $b, c$ are divisible by 4 , then $\operatorname{BU}(M, \phi)=2$ for all $\phi \neq \rho$.

(4) If $\varepsilon=1, \Delta_{1} \equiv 0(\bmod 8)$ and $\Delta_{2} \equiv 2(\bmod 4)$ then $\operatorname{BU}(M, \phi)=2$ for the two epimorphisms $\phi \neq \rho$ such that $\phi^{2}=0$ (that is, that factor through $Z / 4 Z$ ) and $\mathrm{BU}(M, \phi)=3$ for the four such that $\phi^{2} \neq 0$.

(5) If $\varepsilon=1, \Delta_{1} \equiv 4(\bmod 8)$ and $\Theta \equiv-I_{2}(\bmod 4)$ then $\operatorname{BU}(M, \phi)=2$ for all $\phi \neq \rho$.

(6) If $\varepsilon=1$ and $\Delta_{1} \equiv 4(\bmod 8)$, but $\Theta \not \equiv-I_{2}(\bmod 4)$, then $\operatorname{BU}(M, \phi)=2$ for the two epimorphisms $\phi \neq \rho$ such that $\phi^{2}=0$ and $\operatorname{BU}(M, \phi)=3$ for the four such that $\phi^{2} \neq 0$.

(7) If $\varepsilon=-1, \tau \equiv 0(\bmod 4), \Delta_{2} \equiv 2(\bmod 4)$ and $b c \equiv 0(\bmod 8)$ then $\mathrm{BU}(M, \phi)=2$ for the four epimorphisms $\phi \neq \rho$ such that $\phi^{3}=0$ and $\operatorname{BU}(M, \phi)=3$ for the two such that $\phi^{3} \neq 0$.

(8) If $\varepsilon=-1, \tau \equiv 0(\bmod 4), \Delta_{2} \equiv 2(\bmod 4)$ and $b c \equiv 4(\bmod 8)$ then $\mathrm{BU}(M, \phi)=3$ for all $\phi \neq \rho$.

Suppose now that $\pi / \sqrt{\pi} \cong D_{\infty}$. Then the following results are immediate from Section 4.

(9) If $\pi^{\mathrm{ab}} \cong Z / 4 c Z \oplus Z / 4 Z$ then $\mathrm{BU}(M, \phi)=2$ for all $\phi$.

(10) If $\pi^{\mathrm{ab}} \cong Z / 4 c Z \oplus(Z / 2 Z)^{2}$ and $b \equiv 0(\bmod 4)$ then $\mathrm{BU}(M, \phi)=2$ for all $\phi$.

(11) If $\pi^{\mathrm{ab}} \cong Z / 4 c Z \oplus(Z / 2 Z)^{2}$ and $b \equiv 2(\bmod 4)$ then $\mathrm{BU}(M, \phi)=2$ for epimorphisms $\phi$ which factor through $\pi / \sqrt{\pi}$, while $\mathrm{BU}(M, \phi)=3$ otherwise.

\section{Other geometries}

We remark finally that similar arguments may be used to determine the $\mathbb{F}_{2}$ cohomology rings and Borsuk-Ulam invariants for pairs $(N, \phi)$ with $N$ a closed $\mathbb{E}^{3}$ or $\mathbb{N i l}^{3}$-manifold. These manifolds are all Seifert fibred over flat 2 -orbifolds. Since they have been covered in [1], we shall confine ourselves to some brief observations.

The ten closed flat 3-manifolds may be easily treated individually. The only one admitting a class $\phi$ with $\phi^{3} \neq 0$ has group $G_{4}$, with holonomy $Z / 4 Z$ and abelianisation $\mathbb{Z} \oplus Z / 2 Z$. Thus $H^{1}(\pi)=\langle T, X\rangle$, where $T^{2}=0$ and $X^{2} \neq 0$. We may deduce that $T X=0$ also, by mapping $G_{4}$ onto $D_{8}$. It follows easily that

$$
H^{*}\left(G_{4}\right) \cong \mathbb{F}_{2}[T, X, \Omega] /\left(T^{2}, T X, X \Omega, T \Omega+X^{3}, \Omega^{2}\right),
$$

where $\Omega$ has degree two. (Thus $X^{3}=(T+X)^{3} \neq 0$. These classes correspond to the two epimorphisms without integral lifts.)

The possible Seifert bases $B$ of closed $\mathbb{N i l}^{3}$-manifolds are the seven flat 2-orbifolds with no reflector curves: $B=T, K b, S(2,2,2,2), S(2,4,4), S(2,3,6), S(3,3,3)$ or $P(2,2)$. Let $\beta=\pi_{1}^{\text {orb }}(B)$ be the orbifold fundamental group of the base. Then $\pi^{\text {ab }}$ is an extension of $\beta^{\text {ab }}$ by a finite cyclic group $Z / q Z$, if the base is orientable $(B \neq K b$ or $P(2,2))$, and by $Z /(2, q) Z$ otherwise. The ring $H^{*}(\pi)$ depends only on the base $B$ and the residue of $q(\bmod 4)$. 
If $B=T$ or $K b$ then $\pi \cong \mathbb{Z}^{2} \rtimes_{\Theta} \mathbb{Z}$, for some $\Theta \in \mathrm{GL}(2, \mathbb{Z})$. These are in fact the cases requiring most effort. In all other cases $\pi^{\mathrm{ab}}$ is finite, and the projection of $\pi$ onto $\beta$ induces an isomorphism $H_{1}(\pi) \cong H_{1}(\beta)$. When $B=S(2,3,6)$ or $S(3,3,3)$ this group is cyclic. (In particular, such $\mathbb{N i l}^{3}$-manifolds are neither mapping tori nor unions of twisted $I$-bundles.) When $B=S(2,4,4)$ we have $\pi / X^{4}(\pi) \cong \beta / X^{4}(\beta) \cong G_{4} / X^{4}\left(G_{4}\right)$. The cases of $S(2,2,2,2)$ and $P(2,2)$ are related to those of the flat 3-manifolds $G_{2}$ and $B_{4}$, respectively.

The Borsuk-Ulam theorem and its applications and extensions are treated in detail in the book [7].

\section{Acknowledgements}

I would like to thank the organisers of the XVIII Encontro Brasiliero de Topologia for the invitation to their meeting in Aguas de Lindoias, in July 2012, which led to this work, and S. T. Martins for sending me a copy of his $\mathrm{PhD}$ thesis [6]. The use of the extraspecial 2-group $E$ (introduced before Lemma 2.4) was prompted by the work of Carlson on the cohomology rings of 2-groups [2].

\section{References}

[1] A. Bauval, D. L. Gonçalves, C. Hayat and P. Zvengrowski, 'The Borsuk-Ulam Theorem for double coverings of Seifert manifolds', Proc. Brazilian-Polish Topology meeting, July 2012, Proc. Inst. Math. Natl. Akad. Sci. Ukraine, to appear.

[2] J. F. Carlson, 'Cohomology of 2-groups', www.math.uga.edu/ jfs/groups2/cohomology2.html.

[3] D. L. Gonçalves, C. Hayat and P. Zvengrowski, 'The Borsuk-Ulam theorem for manifolds, with applications to dimensions two and three', in Group Actions and Homogeneous Spaces, Fak. Mat. Fyziky Inform. Univ. Komenského, Bratislava (2010), 9-28.

[4] J. A. Hillman, 'The kernel of integral cup product', J. Aust. Math. Soc. 43 (1987), 10-15.

[5] J. A. Hillman, Four-Manifolds, Geometries and Knots, GT Monographs, 5 (Geometry and Topology Publications, 2002); latest revision: see http://www.maths.usyd.edu.au/u/jonh/.

[6] S. T. Martins, Aproximações da diagonal e anéis da cohomologia dos grupos fundamentales das superfícies, de fibrados do toro e de certos grupos virtualmente cíclicos, $\mathrm{PhD}$ Thesis, Universidade de São Paulo, 2012.

[7] J. Matoušek (with A. Björner and G. M. Ziegler). Using the Borsuk-Ulam Theorem, Lectures on Topological Methods in Combinatorics and Geometry (Universitext, Springer, Berlin, 2003).

[8] D. J. S. Robinson, A Course in the Theory of Groups, Graduate Texts in Mathematics, 80 (Springer, New York, 1982).

\section{J. A. HILLMAN, School of Mathematics and Statistics, University of Sydney, NSW 2006, Australia e-mail: jonathan.hillman@sydney.edu.au}

